\title{
Survey Paper on Authenticated Index for Verifiable Cloud Service Selection
}

\author{
Hemavathi H.S ${ }^{1}$, Hamsaveni. $M^{2}$ \\ PG Scholar, Departmentof CSE,VidyaVardhaka College of Engineering, Mysuru, India ${ }^{1}$ \\ Assistant Professor, Departmentof CSE, VidyaVardhaka College of Engineering, Mysuru, India ${ }^{2}$
}

\begin{abstract}
Cloud computing has emerged as a popular paradigm that offers computing resources as scalable of cloud service provider(CSP) and efforts towards intelligent cloud service selection. Cloud brokers as an additional layer to facilitate cloud service selection and services management task for cloud consumer.Although the issue of data privacy can be solved by database encryption techniques. In outsource database model, the cloud broker sever provider query services to user on behalf of the data owner. The user's data are shared and maintained with it concerns of security vulnerability for all the services. this paper survey on how brokerage system provide authenticated CSP's profile to cloud broker and broker to cloud client.
\end{abstract}

Keywords: cloud computing, service selection, cloud brokers, database, brokerage system.

\section{INTRODUCTION}

Cloud computing providing the services over the internet on demand. These services involves network resource, platform, software services, infrastructure software application and virtual servers.

Cloud services offers a scalable variety of storage space and computing capability. These cloud services have different services characteristics, level of abstraction and quality of services, multiple cloud service providers(CSP) available to cloud client for their access. The client may difficult to choose CSP for their requirement and its expensiveness. issues of security and scalability of client data to reduce this problem introduced the another layer is third party as cloud broker. This cloud broker act as monitor and mediation between cloud client and CSP.

The broker collect the information of multiple CSP and list of recommendation to client according to their requirement. The client completely trusted on broker. There is no guarantee of broker provide correctness and completeness of CSP to client. The broker may misbehave with client as well as CSP and recommended his preferable CSP to client. And also cheated to both client and CSP. To overcome this problem we proposed the brokerage system which is mediation between client and CSP by introducing the collector as certificate authority. The collector collect the information of CSP and constructed authenticated CSP's profile and distributed to cloud brokers. Broker sell to client for their requirement . by propose innovative authenticated index structure and verification protocol to allow client to verify the completeness and authenticity of broker's answer using Cloud Service Selection Verification(CSSV) scheme which employs the idea of "separation of duties" to ensure strong security guarantees. This problem is related to that of authentication of query results for outsourced database[6][7].

This paper shows a survey of various Authenticated Index for Verifiable Cloud Service Selection. Section 2 gives Authenticated Index for Verifiable Cloud Service Selection. Section 3 gives conclusion.

\section{RELATED WORK}

Jingwei Li [1] proposed new scheme authenticated index structure and verification protocol to allow client to verify the completeness and authenticity of brokers' answer. This problem is related to that of authentication of query result for outsourced database.

In order to overcome the limitation of existing techniques, both in term of efficiency and supported functionality, they proposed a new authenticated index structure called Multi-Markle $\mathrm{B}^{\text {cloud }}$-tree(MMB ${ }^{\text {cloud }}$-tree), which is a variant of the Merkle $\mathrm{B}^{+}$-tree and is specifically tailored for cloud service selection.

Jianfeng Wang [2] proposed a new verifiable search scheme for dynamic outsourced database, which can achieve verifiable of search result without any pre-computation in data owner side even if the dishonest CSP purposely return an empty set.

The proposed verifiable search scheme of dynamic outsourced database including four entities: data owner, user, cloud service provider(CSP) and arbitrament center(AC).

Sakthivel and Dhiyanesh[3] proposed a method to build a trusted computing environment for cloud computing system by providing secure cross platform in to cloud computing system with message authentication code(MAC). In this 
method some important security services including such as encryption and decryption authentication is checked in cloud computing system.

Zia Ur Rehman[4] proposed the motivation for and importance of having cloud monitoring mechanisms for the current and future inter-operable and federated clouds and propose a novel cloud monitoring approach that collects feedback from users to monitor cloud services in the context of IaaS clouds.

Unlike third party cloud monitoring service, which depends only on benchmarks for cloud performance monitoring, the alternative approach proposed feedback from existing cloud service users for its performance monitoring.

Liu Xin and AnwitamanDatta[5] focused on selecting trustworthy partner to form a good collaborative group. This is a non trivial task because of various reasons. Complexities and conflicts of business interests may make it difficult to find service providers who are willing and able to collaborate.

A service provider who needs to find a partner to provide storage service may be unable to find specific providers may simply reject the collaboration according to their competitive strategies.

MingyanTeng[6] focused on verifying the completeness of query results from the Dataset As a Service(DAS) servers, specifically on multi-dimensional data. Problem can be defined as: given a database D storing massive multidimensional data in a DAS application, a client presents a range query $q$ on the database $\mathrm{D}$ to retrieve all the data within the query range $\mathrm{q}$.

The DAS server returns results $R$ of the query $q$, together with some verification objects $V_{R}$. based on $R$ and $V_{R}$, the client can verify that all the results satisfying $\mathrm{q}$ in $\mathrm{D}$ have been successfully returned and there are no tampered and spurious results in $\mathrm{R}$.

Wang Xiaoming and Lin Yanchun[7] proposed an authentication scheme for users to verify the authenticity and completeness of the query results on a multi-dimensional database. This approach introduce the new concept of Tower Head Node (THN) based on the k-dimensional(k-d) skip list. The approach also shows the development process of an authenticated data structure, the tower-based authenticated skip list(k-d TASL).

This structure supports both multi-dimensional range query and dynamic maintenanve. This approach provide solution to issue related to the authenticity and completeness of query result in the outsourced database model.

Dan Lin [8] proposed a new indexing structure the $\mathrm{B}^{\text {cloud }}$ tree, which is more efficient of storing, indexing and handling updates of a large amount of cloud service information and also efficient query algorithm that supports the generic type of service selection queries. Evaluate this approach using datasets generated based on real scenarios.

Johonnes Harungguan Sianipar et al[9] focus on increasing the cloud consumer trust towards a brokerage system by allowing customer to verify the result of this CSP select.

A consumer should able to verify whether the cloud selection result has given the best CSP based on the customer's requirements. The properties of CSPs should also be verified. Wei Wong [10] proposed a cloud brokerage service to overcome the existing system and address the problem of it. Instead of dealing directly with cloud provider, a customer will purchase from the cloud broker, who has reserved a large pool of instances from IaaS clouds. The cloud brokers pricing gap between reserved and On-demand instances to reduce the expenses of all the users

\section{CONCLUSION}

Thispaper provides a survey on Authenticated index for verifiable cloud service selection. It mainly focused on how cloud client select the cloud service selection by verifying authenticity and completeness using CSSV scheme and study the problem of verifying completeness of query results on multi-dimensional data space

\section{REFERENCES}

[1] J. Li, A. Squicciarini, D. Lin, S. Sundareswaran, and C. Jia. MMBcloud- tree: Authenticated Index for Verifiable Cloud Service Selection. IACR Cryptology ePrint Archive 2015: 379 (2015)

[2] Jianfeng Wang, Xiaofeng Chen, j. Li, "Verifiable Search for Dynamic Outsourced Database in Cloud computing" state key Laboratory Service Networks(ISN), Xidian University, Xi'an, China in 2015 IEEE

[3] Dr. S. Sakthivel, B. Dhiyanesh, "A Privecy-Preseving Storage Security for spatial Data in Dynamics Cloud Environment”in 2013 IEEE

[4] Zia ur Rehman, Omar K. Hussain, Sazia pravin, Farookh K. Hussain, "A Framework for User Feedback Based Cloud Service Monitoring” in 2012 IEEE

[5] Liu Xin, Anwitaman Datta, “ On trust guided collaboration among service providers” in Singapore

[6] Mingyan teng "Verifying the Completeness of query Results on Multi-Dimensional Data" in 2010 on IEEE

[7] Wang Xiaoming, Lin Yanchun, Yu Fang, "Authenticating multi-dimensional query results in outsourced database",in 2015 ISSSN on IET the institute of Engineering and Technilogy.

[8] Dan Lin, Anna Cinzia Squicciarini, Venkata Nagarjuna Dondapati, Smitha Sundareswaran, "A Cloud Brokerage Architecture for Efficient Cloud Service Selection". In 2003 IEEE

[9] Johannes Harungguan Sianipar and Christoph Meinel, “A Verification Mechanism for Cloud Brokerage System”, inISBN: 978-1-4799-1790$7 / 15 / \$ 31.00$ C2015 IEEE

[10] Wei Wang, Student Member, IEEE, Di Niu, Member, IEEE, Ben Liang, Senior Member, IEEE, and Baochun Li, Senior Member, IEEE in IEEE TRANSACTIONS ON PARALLEL AND DISTRIBUTED SYSTEMS, VOL. 26, NO. 6, JUNE 201 